\title{
Anabases
}

ANABASES Traditions et réceptions de l'Antiquité

$10 \mid 2009$

Varia

\section{Le modèle de la silva rerum dans l'écriture du grand reportage par Ryszard Kapuściński}

\section{Magali Soulatges}

\section{(2) OpenEdition}

1 Journals

Édition électronique

URL : http://journals.openedition.org/anabases/728

DOI : 10.4000/anabases.728

ISSN : 2256-9421

Éditeur

E.R.A.S.M.E.

Édition imprimée

Date de publication : 1 octobre 2009

Pagination : 264-273

ISSN : 1774-4296

\section{Référence électronique}

Magali Soulatges, « Le modèle de la silva rerum dans l'écriture du grand reportage par Ryszard

Kapuściński », Anabases [En ligne], 10 | 2009, mis en ligne le 01 octobre 2012, consulté le 21 octobre 2019. URL : http://journals.openedition.org/anabases/728 ; DOI : 10.4000/anabases.728

Ce document a été généré automatiquement le 21 octobre 2019

(c) Anabases 


\title{
Le modèle de la silva rerum dans l'écriture du grand reportage par Ryszard Kapuściński
}

\author{
Magali Soulatges
}

1 Le 23 janvier 2007 disparaît à Varsovie le grand reporter polonais Ryszard Kapuściński. L'hommage, que l'on aurait aimé moins discret, est unanime : avec lui, une page est tournée du journalisme de terrain, dont il aura contribué à entretenir l'aura en sillonnant inlassablement le monde, de l'Asie à l'Amérique du Sud en passant par les anciennes républiques soviétiques, le Moyen-Orient et l'Afrique, où il devient à partir de 1962 l'unique correspondant de l'Agence de presse polonaise PAP. Né en 1932 à Pinsk (actuelle Biélorussie), R. Kapuściński s'était d'abord tourné vers des études d'histoire avant d'entrer dans la carrière journalistique auprès du quotidien de l'Organisation des Jeunesses communistes Sztandar Młodych (L'Étendard des Jeunes), puis pour le magazine Polityka, organe de presse des réformateurs du Parti ouvrier unifié polonais créé en 1957. Témoin d'une trentaine de conflits (« photogéniques ${ }^{1}$ » ou oubliés), coups d'État, révolutions, chutes de dictatures..., il voit ses activités journalistiques restreintes au moment de la loi martiale de décembre 1981, décrétée par le général Jaruzelski face à la montée en puissance de Solidarność, dont il rallie la cause avec d'autres journalistes polonais. Par ses écrits sur les dictatures de Hailé Sélassié (Le Négus, 1978) et du Shah d'Iran (Le Shah ou la Démesure du pouvoir, 1982), il est d'ailleurs suspecté de mettre en cause le régime communiste de son pays ${ }^{2}$.

2 Figure emblématique du journalisme d'investigation, R. Kapuściński bénéficie d'une reconnaissance internationale qui, quoique tardive, lui vaut nombre de récompenses prestigieuses: parmi elles, le prix de la Fondation Principe de Asturias de Comunicación y Humanidades lui est décerné en 2003 pour l'ensemble de ses écrits, et son nom est prononcé à plusieurs reprises pour le Nobel de Littérature. L'œuvre de R. Kapuściński, outre ses papiers rédigés pour différents journaux polonais et étrangers ainsi que des recueils de poésie, comprend plus de vingt ouvrages traduits en plusieurs langues, dont une dizaine seulement en français, les plus marquants de sa carrière ${ }^{3}$. 
C'est Ébène. Aventures africaines, paru en 2000, qui le fait connaître en France du grand public, à la faveur du prix du Meilleur Livre de l'année décerné par la rédaction de Lire ... et d'une incitation quelque peu provocatrice à la lecture :

«Ce n'est [...] pas un livre sur l'Afrique, mais sur quelques hommes de là-bas, sur mes rencontres avec eux, sur le temps que nous avons passé ensemble. Ce continent est trop vaste pour être décrit. C'est un véritable océan, une planète à part, un cosmos hétérogène et immensément riche. Nous disons " Afrique ", mais c'est une simplification sommaire et commode. En réalité, à part la notion géographique, l'Afrique n'existe pas ${ }^{4}$.»

3 Attitude presque « roussellienne " que celle consistant à convoquer des « impressions d'Afrique » pour parler d'un continent qui « n'existe pas »! Le paradoxe toutefois n'est qu'apparent, car chez R. Kapuściński, la « réalité » semble ne jamais exister qu'autant qu'elle se dérobe à une saisie rationnelle et ordonnée, et se construit sur des «choses vues ». Aussi l'exergue d'Ébène peut-elle passer pour une profession de foi ${ }^{5}$. Elle cerne en tout cas de manière assez juste une position à la fois déontologique et méthodologique très personnelle, maintes fois saluée comme la "patte» de ce grand reporter: humilité devant les sujets couverts, approche empathique par la multiplication des points de vue et le refus des itinéraires tracés pour les correspondants d'agence.

Très au-delà des lois du genre - le grand reportage postule par définition l'expression d'une forte subjectivité et sa transcription dans un style personnel marqué -, R. Kapuściński développe une conception de son métier (qu'il apparente à une mission) étayée sur un engagement hissé au rang de ligne de pensée et d'action, et fondant sa méthode sur une hypertrophie de l'observation ${ }^{6}$. Ses témoignages s'offrent ainsi comme autant de leçons, historiques, prétendant moins décrire ou relater, fût-ce avec un regard d'une extrême lucidité critique, que mettre en évidence des processus et des mécanismes interprétables plus largement et plus profondément, à l'échelle de la «longue durée $\mathrm{e}^{7}$ ». Pour rendre compte de la révolution shi'ite qui propulse en 1979 les ayatollahs sur le devant de la scène iranienne, $R$. Kapuściński emprunte par exemple le chemin oblique d'un portrait/biographie du Shah démontrant de manière éloquente comment la "démesure du pouvoir " (sous-titre de l'ouvrage) peut, après en avoir assuré la force, miner une dictature et provoquer sa chute, constat doté d'une dimension universelle dès lors qu'il se lit structurellement comme la confrontation d'une culture ancienne avec un modèle progressiste ${ }^{8}$. "Sorcier du reportage » selon le mot souvent rapporté de John Le Carré à son sujet, R. Kapuściński se disait d'ailleurs formé à l'école des Annales, et un fervent «admirateur de Bloch, Braudel, Fèbvre, de leur manière de penser qui consiste à tenter de construire une image globale à partir de détails et d'extraire de l'histoire des éléments durables, immuables ${ }^{9}$ ». Il n'aura eu de cesse d'appréhender le monde selon cette démarche "anatomique ", et de travailler à une écriture exigeante cherchant à approcher au plus près la ténuité parlante de ces « détails ».

Pour R. Kapuściński, une écriture élaborée s'impose au demeurant comme une nécessité, en prolongement des dépêches d'information rédigées sur le vif et contraintes à la brièveté ${ }^{10}$. Mais ni le genre ni le style de cette écriture, quoique s'inscrivant dans une tradition nationale, ne se satisfont pleinement des modèles scripturaux de la grande école polonaise du reportage qui dans ce pays, a fait accéder le documentaire au rang de véritable genre littéraire, fournissant un arrière-plan esthétique à tout reporter à partir de l'après-guerre ${ }^{11}$. Les hésitations des critiques sont 
fréquentes pour cerner une forme à mi-chemin entre l'enquête et la création, se réclamant à la fois du journalisme et de la littérature, sans être ni l'un ni l'autre. Pour Salman Rushdie, son ami, R. Kapuściński aurait élevé le grand reportage et son écriture au rang d'art ${ }^{12}$, ce que l'Autoportrait d'un reporter paru en 2003 (2008 en France) corrobore en dessinant en creux les contours d'un «art poétique ${ }^{13}$ ». Déroutante parfois, cette poétique semble opter pour une définition minimaliste du grand reportage, se bornant à parler de "texte » et tout aussi vaguement de "livre ", endeçà d'autres termes génériques pourtant plus précis convoqués ailleurs par $\mathrm{R}$. Kapuściński pour caractériser l'originalité de son écriture et la situer dans un champ plus parlant pour le lecteur: "non-fiction writing", "creative non-fiction", " new journalism », "travel littérature », " reportage personnel ", « littérature pédestre », "reportage littéraire», "traduction" (mots renvoyant aussi bien à des catégories reconnues ou des courants du journalisme moderne, notamment anglo-saxons, qu'à un lexique plus personnel) :

"C'est ce que j'écris, des textes ; ce mot reflète mon type d'écriture de la manière la plus précise. Je ne suis pas un écrivain de fiction ni un journaliste de presse. J'écris mes textes, mon genre littéraire, ma littérature. [...]

Donc, qu'est-ce que j'écris? J'écris des textes. Je suis incapable de donner une autre définition à mon écriture : c'est la définition qui me correspond le mieux ${ }^{14}$.»

6 Cependant, corrélé à d'autres affirmations et rapporté à la forme même des œuvres de R. Kapuściński, un terme presque intrus dans ce champ sémantique, silva rerum, apparaît au détour des entretiens qui composent cet "autoportrait ", dévoilant des soubassements plus anciens, en même temps que plus rares, de cette écriture :

«Parfois en décrivant ce que je fais, j'ai recours à l'expression latine silva rerum, une «forêt de choses». C'est mon univers, une forêt de choses, et je vis en voyageant dedans. Pour comprendre le monde, il faut le pénétrer aussi profondément que possible ${ }^{15}$.»

7 «Expression" ne signifie bien sûr pas modèle, et il est peu probable que le référent antique soit ici directement convoqué, qu'il s'agisse du genre poétique de la "silve", illustré par Stace, ou des silvae rerum de la rhétorique. En revanche, cet héritage antique vient naturellement s'inscrire dans la culture polonaise qui, férue d'Italie, s'ouvre aux $\mathrm{XV}^{\mathrm{e}}$ et $\mathrm{XVI}^{\mathrm{e}}$ siècles aux idées de l'Humanisme et dans ce contexte, cède à la mode des silvae qui se développe en Europe à partir de la Renaissance. Mode qu'inspire donc la redécouverte savante des Lettres et de la culture oratoire antiques, dans lesquelles les humanistes trouvent un écho à leur propre inclination pour la copia et la varietas de l'érudition. Plusieurs influences se conjuguent ici : elles viennent de la poésie, de la rhétorique et de la philosophie. L'imitation des Silvae statiennes, perçues comme un genre poétique « nouveau » dégagé des canons esthétiques et révélant une subjectivité, justifie une prolifération de recueils de poésie néo-latine de circonstance s'appropriant les principes esthétiques de l'œuvre antique (liberté de ton et de composition, poétique de l'impromptu, illusion du naturel), au-delà du seul titre ${ }^{16}$. Du côté de la rhétorique, c'est dans le De Oratore de Cicéron et le De Institutione oratoria de Quintilien, qui répercute les préceptes cicéroniens, que se niche la silva: tandis que le premier texte recommande d'" amasser un ample fonds de choses et d'idées » (VIII, 26 : primum silva rerum ac sententiam comparanda est $)^{17}$, le second, théorisant l'imitation et l'improvisation, met en avant l'« abondance des idées et des mots » $(\mathrm{X}, 1$ : copia rerum ac verborum) dans laquelle les orateurs doivent puiser leur matière, "qu'ils appellent une silve» (X, 3: hanc silvam vocant $)^{18}$. Enfin, la démarche de l'anthologie ou des "florilèges", qui caractérise la pratique de certaines écoles philosophiques de 
l'Antiquité, se retrouve dans la promotion humaniste des recueils de "mélanges" rêvant d'embrasser le vaste monde dans son foisonnement et sa diversité. De toutes ces continuités à l'œuvre dans la silva rerum humaniste, on voit bien que ressort l'idée d'une hétérogénéité construite au fil du temps et dispensée d'une mise en ordre, à l'image de l'expérience ouverte du monde qui s'impose à ce moment-là : quelque chose comme une métonymie de la bibliothèque humaniste idéale, in-terminable et mettant en valeur le collectionneur à travers la collection ("C'est mon univers, une forêt de choses, et je vis en voyageant dedans. » [nous soulignons]). D'où, de la Renaissance au $\mathrm{XVIII}^{\mathrm{e}}$ siècle, une évolution des silvae de l'inventaire à dominante poétique à des recueils de plus en plus éclectiques, « choses mêlées » réunissant et entrecroisant divers types de textes, genres, thèmes, tons, etc. - le nom de miscellanea vient alors parfois concurrencer celui de silvae.

Or cette évolution aboutit en Pologne, aux XVII ${ }^{e}$ et XVIII ${ }^{\mathrm{e}}$ siècles, à une forme très particulière de la silva, véritable phénomène littéraire local, qui selon toute vraisemblance sous-tend la mention par R. Kapuściński de l'expression latine ${ }^{19}$. Documents de la noblesse polonaise, similaires à des registres, les silvae rerum sont des recueils de textes indépendants, pour la plupart manuscrits - quelques fragments imprimés, tirés d'almanachs, y figurent parfois -, sans rapport apparent entre eux, et collationnés selon des intentions diverses, irréductibles à une démarche a priori esthétique. Tout en étant placées sous le signe de l'hétéroclite, les pièces constitutives de ces " cahiers" factices ressortissent à trois grands types de contenus, non hiérarchisés : politique (discours, lettres officielles, journaux des diètes et diétines...), économique (feuillets de comptes, listes de dépenses et de revenus...), "moral» (sentences, suppliques à l'occasion de maladies...), à quoi s'ajoutent des notules privées enregistrant petits et grands événements de la vie familiale (naissances, mariages, deuils) ou attestant une généalogie, ainsi que quelques pièces littéraires, de poésie le plus souvent. Réunis au hasard des circonstances ou des souvenirs, et voués à être transmis ou partagés, ces mélanges répondent à une forme souple et ouverte. Pris dans l'économie manuscrite, ils circulent entre générations d'une lignée et/ou entre amis, qui les recopient, amendent, raturent, coupent, augmentent, commentent, révisent, réagencent, etc.; en ce sens, ils sont un support privilégié de la sociabilité, avec des modalités d'appropriation qui témoignent à la fois des réseaux dans lesquels ils circulent et de la distance prise à certains moments par rapport à ceux-ci. Un protocole prévoit ainsi que tout emprunt sera dûment indiqué, par une formule canonique portée au frontispice des copies, signe d'une volonté de reconnaissance auctoriale qui s'explique autant par une démarche personnelle - la "forêt de choses " reflète les intérêts et la curiosité intellectuelle particuliers de celui qui la compose - que par une intention collective. À travers les silvae rerum en effet, c'est cette noblesse polonaise si singulière (la szlachta) qui s'exhibe comme en un miroir et se dit ad posteritatem ${ }^{20}$, porteuse des valeurs d'une " polonité » qu'elle prétend tirer de ses mythiques origines sarmates : supériorité, héroïsme, indépendance vis-à-vis de tout pouvoir (la "Liberté d'or »), mais aussi conservatisme, intolérance religieuse et xénophobie. Aux côtés de leur fonction archivistique, donc mémorielle, les silvae rerum nobiliaires polonaises des $\mathrm{XVII}^{\mathrm{e}}$ et $\mathrm{XVIII}^{\mathrm{e}}$ siècles remplissent ainsi une fonction identitaire, qui tend certes à l'hagiographie d'un groupe social particulier et restreint, mais intéresse surtout ici par la démarche qui lui est associée : celle de la collection, caractérisée par une forme et une intention spécifiques lui conférant le statut d'un "genre». Genre au fond de l'ordre de l'essai ${ }^{21}$, comme le disent aussi à leur manière les autres noms, latins ou 
polonais, parfois portés par ces silvae : vorago rerum (" gouffre des choses»), notata varia ( " notes variées »), nihil et omnia (" tout et rien »), arka noego ("l'Arche de Noé »), ogród kwiatów (« le jardin des fleurs»), r"kopis rzeczy róænych («manuscrit des choses diverses »)...

9 Ancrés dans leur culture polonaise ${ }^{22}$, les « textes » de R. Kapuściński offrent bien des similitudes avec la forme et l'esprit de ces silvae rerum nobiliaires. Acquis à la varietas, ils relèvent autant de genres conventionnels tels le commentaire ou l'essai politique, le récit, le portrait, la biographie, que de types mineurs comme l'anecdote, la remarque, la note, la saynète, l'esquisse, etc., toujours mêlés au sein d'un même ouvrage ; s'y ajoute, au moment de leur rassemblement en un livre, un paratexte de cartes et citations en épigraphe. Registres et tonalités sont aussi divers: descriptif, narratif, didactique, grave, ironique... "La curiosité est un trait de caractère du reporter », explique R. Kapuściński, estimant être de ces « gens peu nombreux [...] qui ont besoin de connaître le monde dans sa diversitéé ${ }^{23}$. " Enfin, leur premier mode de publication sous forme de chroniques «en feuilletons ${ }^{24}$ » les rapproche de l'esprit de la collection patiemment constituée au fil du temps. Réunis en recueils parfois conçus comme des dossiers (à charge, dans le cas du Négus ou du Shah), ces textes s'organisent selon des configurations différentes qui n'ont de la simple juxtaposition que l'apparence. Outre sa cohérence thématique, signifiée a minima par les titres, chaque ouvrage en effet semble comme explorer et mettre en valeur une formule possible de la silva rerum, une modalité particulière du genre en adéquation avec le sujet.

10 Le Négus, qui relate à travers un portrait du dictateur la fin du régime éthiopien de Haiilé Sélassié, joue sur la polyphonie et la rupture en entrecroisant les « descriptions de ceux qui avaient fréquenté les salles, les bureaux, les corridors du Palais ${ }^{25}$ » (discours directs attribués sous de simples initiales) et le propre discours de R. Kapuściński, identifié par une typographie en italiques. En épigraphe des trois grands volets de cette chronique linéaire d'une mort annoncée ("Le Trône», "Ça va, ça vient", "L'Effondrement »), sont insérées plusieurs citations qui démultiplient encore les voix, jusqu'à la pirouette finale de l'ouvrage, qui clôt celui-ci sur une double dépêche de l'AFP et de l'Ethiopian Herald annonçant la mort du dictateur - toutes les voix convoquées jusque-là se taisent ainsi, comme disparaît le régime de Sélassié, laissant la place à la voix anonyme, uniquement informative, des agences de presse.

11 Le Shah repose sur une même structure en triptyque ("Cartes, visages, champs de fleurs ", "Daguerréotypes", "La flamme morte»), mais ses volets sont articulés différemment : le premier fournit une sorte d'avant-propos décrivant la situation du reporter (et partant, les conditions de son enquête) et le troisième livre les résultats de celle-ci en opérant une synthèse élargie sur les « causes des révolutions ", que le second volet a nourrie et préparée par une succession (faussement) aléatoire d'observations, clichés et notes, et l'écoute de conversations enregistrées tout au long de l'enquête menée.

12 Imperium, que précèdent neuf citations en épigraphe, une carte géographique et un avant-propos de l'auteur, extrapole la formule du Négus aux dimensions démesurées du territoire de l'ancienne Union soviétique. L'ouvrage reprend l'idée d'une forme se délitant au fil du propos, en passant d'une narration suivie à un « recueil de réflexions, remarques et notes, nées de mes voyages, conversations et lectures", à l'image de l'effondrement du régime communiste. La chronologie y joue donc un rôle important les trois parties du livre découpent trois grandes périodes de reportage -, bien que 
brouillée dans les pages par un jeu de polyphonie et de résurgences de "personnages, lieux et intrigues ${ }^{26} »$.

Les «aventures africaines " d'Ébène présentent en un kaléidoscope d'expériences diverses quarante années passées à arpenter un continent insaisissable, au moyen d'une écriture de l'impromptu s'accordant par sa liberté de ton à la modestie de l'intention et de rencontres hors des «itinéraires officiels, [des] palais, [des] hommes importants et [de] la grande politique ${ }^{27} »$. Sous des titres variés et en eux-mêmes peu éloquents («Le cœur du cobra», " Les cristaux noirs de la nuit »...), les vingt-neuf textes pour la plupart non datés rassemblés dans cet ouvrage s'offrent comme autant de pièces d'un puzzle voué à l'incomplétude, a fortiori à une impossible clôture. C'est peut-être de tous les livres de R. Kapuściński, celui qui illustre le plus ostensiblement par sa structure pourtant la plus simple (une juxtaposition de textes non hiérarchisée), l'humble conviction du reporter, historien de formation, qu' « aujourd'hui, tout livre sur l'actualité ne peut être qu'un texte ouvert, le premier tome d'un texte virtuel. L'histoire poursuivra la rédaction des tomes suivants, et leurs auteurs peuvent être des personnes complètement différentes. Nous devons accepter l'idée d'écrire des livres inachevés ${ }^{28} »$.

Il n'y aura pas de paradis (réédition en 1990, sous un nouveau titre, de l'ouvrage de 1986, D'une guerre l'autre) relève encore de la silva rerum, tout en étant, de l'aveu même de R. Kapuściński, une œuvre un peu à part. La copia y semble posée d'emblée comme un postulat, avec ce sous-titre : La guerre du foot et autres guerres et aventures ${ }^{29}$, annonçant l'inventaire. Elle débouche sur une architecture sophistiquée du livre où s'entremêlent, selon un ordre pensé avec des équilibres en répons, des textes en majorité datés (de 1960 à 1976) et des considérations personnelles tendant à l'autobiographie - celles-ci apparaissent surtout dans le "plan d'un livre qui aurait pu commencer à cet endroit (ou petits soucis dont je n'ai jamais parlé)». Chaque grande partie constitutive (reportages, "plan du livre » non écrit) est elle-même régie par des jeux et des règles manifestement prémédités: tandis que la chronologie des reportages dessine un agencement dont « la guerre du foot » constitue la clef de voûte, le livre virtuel voit son plan progressivement envahi par des développements niant son caractère d'ébauche et ruinant son unité idéale - tout semble in fine se résoudre en un empilement d'anecdotes. Au fil des pages, le livre phagocyte d'ailleurs de plus en plus le reportage, tout en se révélant de plus en plus difficile à écrire. L'ironie règne ici en maîtresse, et entre en tension vertigineuse avec des sujets traités selon une tout autre gravité. Textegigogne, le «Plan d'un livre qui aurait pu commencer à cet endroit » se déploie en une "Suite du plan d'un livre qui aurait pu commencer, etc. ", puis une "Suite du plan d'un livre jamais écrit qui aurait pu l'être... », suivie d'un " Il est grand temps de commencer à écrire le livre suivant jamais écrit ", que prolonge, à deux reprises, une « Suite de " Il est grand temps de », ou suite du plan d'un autre livre jamais écrit qui, etc. », laquelle s'achève, et achève le livre, sur un cocasse "Et pourtant, Ishmael vogue toujours ". C'est de tous ces tiroirs ouverts que semblent sortir, comme de la boîte de Pandore, les rudes textes assumant en regard le statut de reportages.

«D'une guerre l'autre est un livre existentiel, complètement différent des autres. Il traite du phénomène de désarroi, de la difficulté à se retrouver dans divers contextes. De l'incapacité à définir, trouver, comprendre. [...] Ce livre parle d'un pays où personne ne sait ce qui va advenir demain, dans un mois, dans une semaine, qui va prendre le pouvoir, qui se battra contre qui. Le doute, voilà le thème de ce livre $^{30}$." 
Dans la silva rerum, R. Kapuściński trouve donc bien une forme esthétique à la mesure de son projet d'écriture du grand reportage, quand celui-ci entend "pénétrer aussi profondément que possible » la "forêt des choses » par quoi se dit le monde. Au sein de cette forme, chaque texte se fait alors fragment, détail d'un vaste ensemble en soi déjà morcelé et mobile, gouverné par les lois du foisonnement disparate et de la variété (copia et varietas) $^{31}$. Parce qu'insaisissable, ce monde ne peut au mieux se raconter qu'à travers des agencements provisoires de ses divers éclats. Si la silva rerum fournit le modèle historique et littéraire de ces configurations ponctuelles, l'on pourrait aussi bien invoquer le modèle pictural du collage, dont R. Kapuściński se réclame d'ailleurs autant que de la silva. Ces deux formes esthétiques, très proches, favorisent en effet à ses yeux l'expression spontanée et toute en finesse, car en creux, de la subjectivité :

« La forme du collage en peinture correspond vraiment le mieux aux textes que je crée. Autrement dit, je choisis différentes techniques, différents objets et, sans réfléchir à leur classification (je laisse cela aux critiques), je les utilise de façon qu'ils expriment au mieux ma pensée. Pourquoi ai-je écrit tel ou tel texte en prose? Pourquoi est-ce que j'écris des poèmes ? Parce qu'il y a des choses qui ne peuvent être exprimées autrement. Je ne me demande pas alors s'il s'agit d'un reportage, d'un essai ou d'un poème "purs" (au sens de la définition classique). Et tout ce que j'écris, je l'écris à travers moi-même ${ }^{32}$. "

De toute évidence, chez le grand reporter polonais, cette écriture élaborée et exigeante rencontre une position éthique mise au service d'un journalisme ambitieux et libre, dans lequel l'expérience du reportage cherche toujours à déborder la conscience individuelle pour accéder à une autre hauteur de vue sur le monde, dont il s'agit de mettre au jour les mécanismes profonds, comme l'illustrent et le glosent magistralement les six tomes de Lapidarium, immense silva absorbant toutes les autres. Partant, la leçon de l'œuvre de R. Kapuściński est aussi philosophique, pour ne pas dire métaphysique. Faisant de l'effort un préalable nécessaire à la connaissance ${ }^{33}$, elle s'offre comme une ascèse, tournée in fine vers l'Autre, i.e. ce lecteur qu'elle entend accompagner, guider peut-être, dans la "forêt des choses ", de la même manière que les auteurs de silvae indiquaient, aux $\mathrm{XVII}^{\mathrm{e}}$ et $\mathrm{XVIII}^{\mathrm{e}}$ siècles, des parcours possibles dans leurs recueils en ménageant renvois, index et tables des matières. De cette foi qu'on pourrait dire « humaniste » en l'écriture, R. Kapuściński ne s'est jamais départi : « Dans ma vie, j'ai toujours essayé de me tenir à un triple principe : ni titres, ni postes, ni fonctions. J'estime que je dois tout simplement écrire. Tout ce qui n'est pas écriture, tout ce qui ne sert pas l'écriture, je le considère comme du temps perdu. »

\section{NOTES}

1. Autoportrait d'un reporter, Plon, « Feux croisés », 2008 pour la traduction française, p. 137.

2. La lumière n'est pas faite sur une éventuelle « double » carrière de R. Kapuściński, de 1965 à 1972 ou 1977, en tant qu'agent des services secrets polonais. 
3. En attendant la traduction en français des six volumes de Lapidarium (I-VI, Varsovie, Czytelnik, 1990-2006), impressionnante somme de notes et fragments de toute une vie de reportages, dépassant en ampleur toutes ses œuvres publiées.

4. Avertissement d'Ébène, Plon, « Pocket ", 2000 (édition originale, 1998).

5. Voir aussi l'Avant-propos d'Imperium (1993 ; Plon, « Feux croisés », 1994 pour la traduction française).

6. Une « anecdote " du Shah (Le Shah ou la Démesure du pouvoir, Flammarion, « 10/18 ", 1986 pour la traduction française, p. 143) résume bien ce postulat : « Ce n'est pas uniquement pour le plaisir des sens que je viens voir ce tableau de couleurs [la devanture d'un marchand d'épices de Téhéran, décrite précédemment]. Le destin quotidien de l'étalage est aussi une source de renseignements sur les futurs événements politiques, car Engelob est le boulevard des manifestations. S'il n'y a pas d'étalage sur le trottoir le matin, c'est que l'Arménien s'attend à une journée chaude - il y aura une manifestation. Il préfère cacher ses fruits et ses épices plutôt que de les laisser piétiner par la foule. Cela signifie également que je dois me mettre au travail et déterminer qui va manifester, et pour quoi. Si en revanche je vois la palette bigarrée de l'Arménien briller de loin dans la rue Engelob, alors je sais que ce sera une journée ordinaire, paisible, sans événements et que je peux aller boire un whisky chez Léon, la conscience tranquille. "

7. De l'éditeur du Négus (1978 ; Flammarion, 1984 pour l'édition française), au-delà de l'éloge éditorial de rigueur dans une quatrième de couverture : "Mort et folie rôdent dans cette chronique qui prend l'ampleur d'une fable cruelle et captivante, où l'histoire se fait aussi exemplaire qu'une légende. "

8. Le portrait joue souvent chez R. Kapuściński un rôle d'épiphénomène condensant la leçon politique en un symbole. Pour donner un autre exemple : « J'ai naguère pensé écrire un livre sur Amin, car il symbolise l'alliance du crime et de l'absence de culture » (« Amin » [portrait d'Amin Dada], dans Ébène).

9. Autoportrait, p. 21. Quand le reportage s'appuie souvent sur du factuel, ancré dans l'actualité, celui pratiqué par R. Kapuściński se montre plutôt avare en dates, chronologies précises, même si des rappels historiques viennent s'insérer naturellement dans le propos.

10. Autoportrait, p. 35.

11. Cf. M. CARLIER dir., La Vie est un reportage. Anthologie du reportage littéraire polonais, Montricher, Éditions Noir sur Blanc, 2005. R. Kapuściński se réclame explicitement des grands noms de cette école née avant la Seconde Guerre mondiale, et très active dans les années 1960 : K. Pruszyński et M. Wańkowicz, ainsi que M. Brandys, à l'origine de l'« École des trois K » (H. Krall, K. Kakolewski et Kapuściński lui-même).

12. Cf. C. WAUTHIER, «Dans l'Afrique profonde » [à propos d'Ébène], Le Monde Diplomatique, janvier 2001, p. 27.

13. Autoportrait d'un reporter rassemble de manière souple des extraits d'entretiens et de conférences données à diverses occasions par R. Kapuściński entre 1985 et 2002. La logique biographique passe cependant au second plan d'une composition en cinq chapitres portant sur « la carrière du grand reporter polonais, sa conception du métier, la spécificité de son écriture, sa vision du monde et des médias ", et tendant plutôt à l'essai " philosophique ", dans l'esprit de Mes Voyages avec Hérodote (2004 ; Plon, « Feux croisés », 2006 pour la traduction française).

14. Autoportrait, p. 80 et p. 92.

15. Autoportrait, p. 93.

16. En Pologne par exemple, Jan Dantyszek, grande figure de l'Humanisme, auteur de nombreuses poésies de circonstance, se signale par une De nostrorum temporum calamitatibus Silva (1530) qui dépasse les thématiques habituelles du genre en proposant un tableau politique et religieux des attaques turques contre la chrétienté. Sur le courant de la « silve » à la Renaissance, voir P. GALAND-HALLYN et F. HALLYN, «"Recueillir des brouillards" : éthique de la silve 
et poétique du manuscrit trouvé ", in Le Poète et son Euvre. De la composition à la publication, études réunies par J.-E. Girot, Droz, 2004. Sont encore à paraître les actes du colloque qui s'est tenu du 2 au 5 juillet 2008 à l'Académie royale de langue et littérature néerlandaise de Gand : « La "silve" : histoire d'une écriture libérée en Europe de l'Antiquité au XVIII ${ }^{\mathrm{e}}$ siècle ».

17. De oratore, trad. E. Courbaud, Les Belles Lettres, 1967.

18. De Institutione oratoria, $\mathrm{X}, 7$, trad. J. Cousin, Les Belles Lettres, 1979.

19. Cf. S. RosZAK, "Silvae rerum. Les manuscrits de la noblesse polonaise des XVII et XVIII siècles ", in J. DUMANOWSKI ; M. FIGEAC (dir.), Noblesse française et noblesse polonaise. Mémoire, identité, culture $X V I^{e}-X X^{e}$ siècles, Pessac, Maison des Sciences de l'Homme d'Aquitaine, 2006, p. 357-369.

20. Formule fréquemment consignée en tête des silvae, et qui en souligne la perspective mémorielle.

21. Une résurgence du modèle des silvae rerum dans le roman polonais depuis les années 1990 est précisément interprétée comme une tendance du genre romanesque à l'« essaiisation ", dans un contexte moderne et postmoderne où la littérature renoue avec les poétiques du fragment (cf. P. CZAPLIŃSKI, «Défi de la prose polonaise des années 90 ", http://www.culture.pl/fr/culture/ artykuly/es_proza_lat_90).

22. Autoportrait, passim, mais plus particulièrement p. $53:$ : [...] pour écrire un texte en polonais, il faut lire Żeromski, Prus, Nałkowska, les belles lettres polonaises qui nous ramènent au sein de nos représentations, de notre vocabulaire, et permettent de nous "installer" dans le genre. "

23. Autoportrait, p. 13-14, et infra, p. 272-273.

24. Autoportrait, p. 105-106.

25. Le Négus, p. 21.

26. Imperium, Avant-propos.

27. Ébène, Avertissement.

28. Autoportrait, p. 21.

29. « La guerre du foot » parle du match retour de qualification pour la Coupe du Monde de 1969 entre le Honduras et le Salvador, dont les résultats déclenchèrent un conflit entre les deux pays à l'origine de six mille morts et plus de quinze mille blessés.

30. Autoportrait, p. 99.

31. En prolongement presque naturel de cette écriture du fragment, illustrative d'un monde impossible à décrire dans sa globalité, le style se veut lui-même mimétique. Pour rédiger Le Shah, le recours au «polonais ancien » s'est imposé : «J'avais besoin d'une langue archaïque pour montrer la nature archaïque de l'autoritarisme »; et pour Imperium, il a fallu « étirer [la phrase] pour qu'elle puisse embrasser la grandeur du thème, l'immensité des espaces et l'insaisissable lenteur de ce pays. " (Autoportrait, p. 82-83).

32. Autoportrait, p. 92.

33. Autoportrait, passim, mais notamment p. 17.

\section{AUTEUR}

\section{MAGALI SOULATGES}

Université d'Avignon et des Pays de Vaucluse

magali.soulatges@univ-avignon.fr 